\title{
How do I dilate a benign esophageal stricture?
}

\author{
Nitin Khanna MD FRCPC
}

$\mathrm{T}$ he management of a benign esophageal stricture is a problem encountered frequently by the endoscopist. Dilation of an esophageal stricture is a procedure that can be quite satisfying due to the prompt resolution of debilitating symptoms experienced by patients. However, with the potential for very serious complications, in particular, esophageal perforation, it is a procedure that can produce significant anxiety. There has been a relative lack of updated clinical guidelines for practicing endoscopists to follow, and it is an area with a variety of techniques that can be used. The purpose of the present article is to review my approach to performing the dilation of a benign esophageal stricture and to hopefully provide some clarification regarding some of its myths. I will not focus on the management of malignant strictures, which is a topic with enough issues for a separate review.

Long-standing gastroesophageal reflux disease is the cause of the majority of benign strictures in the esophagus (1). These 'peptic

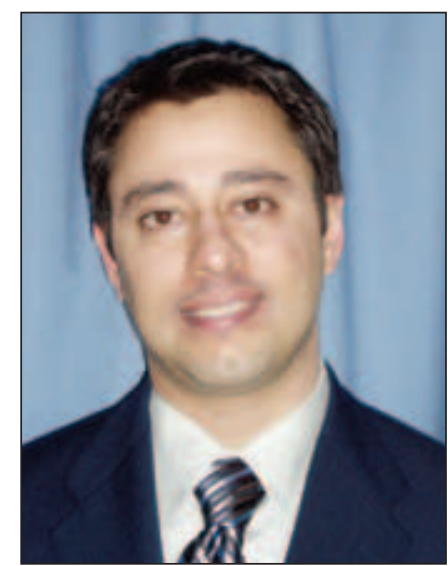

Dr Nitin Khanna essential for the dilation of long or high-grade strictures. Typically, it is most efficient to perform endoscopy and dilation together in one session.

There are two broad categories of dilators: mechanical (push-type or bougie) and balloon. Both types may or may not be used with a guidewire. Mechanical dilators can either be passed freely or over a guidewire. The Maloney (Medovations, USA) is the most commonly used bougie dilator. Made of rubber and filled with mercury or tungsten, it has a tapered tip and is freely passed without a guidewire. The Savary-Gilliard (Wilson-Cook, USA) is a tapered thermoplastic dilator and is passed over a guidewire. These push-type dilators come in a variety of sizes. Less commonly used older systems include the rounded tip dilator (Hurst, Medovations, USA) and the wireguided metal olive-tip dilator (Eder-Puestow, Eder Instruments Company, USA). Dilation of the stricture occurs by both longitudinal strictures' usually occur in the distal esophagus and are typically short in length. Other relatively common causes of benign esophageal strictures include Schatzki ring, esophageal web and strictures as a result of external beam radiation, sclerotherapy, caustic ingestion and surgical anastomosis. Malignancy always needs to be considered upon the diagnosis of an esophageal stricture, with the most common primary cancers including squamous cell carcinoma and adenocarcinoma (2). Infiltration of the esophagus from other mediastinal malignancies and metastatic disease can also rarely occur.

Dysphagia is the cardinal symptom of esophageal obstruction. Most patients with a mechanical cause for their dysphagia will have the most difficulty swallowing solid food. A preceding history of gastroesophageal reflux disease raises the suspicion of a peptic stricture. Nonprogressive, intermittent, solid food dysphagia is most indicative of a Schatzki ring or esophageal web. The new onset of dysphagia, progressively worsening dysphagia, with or without other warning symptoms such as weight loss and bleeding, raises the concern of underlying malignancy. Diagnosis is achieved by barium swallow and endoscopy. Barium swallow can be helpful to confirm a stricture and to establish the lumen diameter, location and length of the stricture. Other structural abnormalities of the esophagus such as a diverticulum or hiatus hernia may also be diagnosed. For physicians with long wait times, a barium swallow can help in the triaging of patients with high-grade strictures or features suspicious for malignancy more urgently. Endoscopy has the advantage of tissue acquisition for pathology and is and radial force, and occurs progressively as the larger part of the dilator passes across the stricture (3). Balloon dilators are passed through the scope (TTS). Available in a variety of diameters and lengths, dilation occurs by radial force as the balloon is expanded. Newer versions of the TTS balloon dilator (Boston Scientific Corp, USA) have guidewire capability and enable expansion to three different sizes at $1.5 \mathrm{~mm}$ increments without changing balloons.

Before performing esophageal dilation, the patient's history should be reviewed and a physical examination performed. Informed consent should be obtained with explanation of the potential complications, such as the side effects of sedation, perforation, hemorrhage, aspiration and chest pain. Premedication with antibiotics needs to be considered as per current guidelines, given the risk of transient bacteremia $(4,5)$. Appropriate measures should be taken for patients using antiplatelet agents and for those who are anticoagulated. I recommend discontinuation of acetylsalicylic acid and Plavix (Sanofi-Synthelabo Canada Inc) for one week before the dilation and aim for an international normalized ratio of less than 1.3 for patients on Coumadin (Bristol-Myers Squibb Canada).

Esophageal dilation is usually performed as an ambulatory outpatient procedure using conscious sedation and topical anesthetic of the oropharynx. With a tight stricture, one should anticipate a longer procedure time and more discomfort to the patient than a typical diagnostic upper endoscopy. Thus, patients may require more sedation and the addition of an analgesic agent. Sedation may not be required at all for a 
straightforward bougie dilation in patients undergoing repeated procedures, and sufficient oropharyngeal topical anesthesia using a $1 \%$ lidocaine solution spray or gargle is often all that is required. The procedure is usually performed in the left lateral decubitus position; however, unsedated patients undergoing bougie dilation with a Maloney can be placed in the seated position.

Patients should be fasting. If there is suspicion of a high degree of esophageal obstruction, consideration should be given to a longer period without solid food intake preceding endoscopy to ensure that the esophagus is clear of food. A $24 \mathrm{~h}$ period on clear fluids, and fasting for $4 \mathrm{~h}$ would be a reasonable preprocedure preparation routine if possible. This should decrease the risk of pulmonary aspiration and improve endoscopic visualization of the esophagus during the procedure.

The first decision that needs to be made is the selection of the type of dilator that is going to be used. Maloney bougie dilation is appropriate for single, distal strictures where the internal diameter of the lumen at the stricture site is greater than $10 \mathrm{~mm}$ and can be traversed by an endoscope. These are typically peptic strictures and Schatzki rings. The use of a guidewire or balloon is not required in these situations (5). When the stricture is tight, tortuous or long, the Maloney bougie may not pass into the stomach and the tip of the dilator may catch on the stricture or esophageal mucosa, causing a perforation. Similarly, when strictures are associated with a large hiatus hernia, esophageal diverticulum or tracheoesophageal fistula, a Maloney should not be used. In these circumstances, the use of a guidewire or TTS balloon will improve the success of the dilation and decrease the risk of perforation (6).

There are other techniques reported in the literature but are not ready for prime time, given a lack of evidence. These include the injection of corticosteroids into the stricture and temporary nonmetal stents $(7,8)$. These have primarily been reported for use in the setting of refractory strictures.

When using a guidewire, it is preferable to pass the wire down to the gastric antrum or duodenum for stability. Fluoroscopy would be required to confirm this if the endoscope does not traverse the stricture. There have been conflicting reports regarding the advantage of using fluoroscopy and efficacy may be more related to the endoscopist's experience (9-12). Given the limits of the availability of fluoroscopy, endoscopists may need to pass the guidewire carefully down the lumen using endoscopic visualization of the proximal end of the stricture and feeling for any resistance (13). There is a lack of evidence regarding efficacy and complication rates of this technique without fluoroscopy in the literature. The esophagus should be dilated progressively and carefully up to the point that the endoscope can be passed into the stomach to confirm guidewire positioning. If the guidewire does not pass easily and there is any doubt about its position, the esophagus should NOT be dilated. Both the Savary dilator and TTS balloon can be used with preference based on experience and the endoscopist's level of comfort.

It is important to remember that most strictures such as peptic strictures have developed over months to years, and restoration of normal lumen diameter cannot be rushed. The size of dilator that should be used is estimated endoscopically by comparing the lumen with the diameter of the endoscope. The first dilation performed should be approximately the diameter of the stricture. Dilation is considered to have been performed when there is a moderate or significant amount of resistance. If there is no resistance, dilation has not been performed. Excessive force is not necessary and should not be used. It is reasonable to follow a 'rule of three', performing up to three dilations that meet resistance per session, with an increase in the stricture diameter by $2 \mathrm{~mm}(6 \mathrm{Fr})(14,15)$. It may be necessary to perform fewer dilations during a session if the stricture is very tight, the patient experiences significant pain or there is evidence of bleeding.

When to repeat dilation depends on the degree of stenosis and success of the initial procedure. The procedure should be repeated within one to two weeks for high-grade strictures. If there is a relatively short interval between sessions, the endoscopist may be able to begin the next series of dilations with the size that was last used the previous time. A smaller size may be needed if there is a delay between sessions. Also, there can be shrinkage of strictures after dilation which is difficult to predict. High-grade strictures will have to be reassessed endoscopically anyway, and the choice of dilator size that will be used first can be estimated based on the endoscopic appearance. The timing of subsequent dilations will depend on how the patient does clinically. Before proton pump inhibitors, greater than $50 \%$ of patients required repeat dilation even after the resolution of dysphagia following dilation of a peptic stricture within the first year $(16,17)$. Proton pump inhibitors appear to decrease the need for repeat dilations in patients with a peptic stricture undergoing dilation (18). However, despite adequate dilation and acid suppression, some patients need to be brought back on a regular basis (eg, every three months) for dilation.

Endoscopists likely receive less training in the use of TTS balloon dilation. Comparison with mechanical dilation suggests equal efficacy and safety in the dilation of benign esophageal strictures (19). The optimal number of dilations per session remains unknown. The size of balloon to be used initially should be approximately the diameter of the stricture or slightly larger. I perform up to three dilations in one session holding the balloon inflated for $30 \mathrm{~s}$ to $60 \mathrm{~s}$ using an increasingly larger diameter. The ability to pass the inflated balloon through the stricture helps to confirm successful dilation. If fluoroscopy is used with balloon dilation, the balloon is inflated using diluted water-soluble contrast. Correct positioning and successful dilation are confirmed fluoroscopically by the presence and obliteration of the 'waist' caused by the stricture on the balloon.

The end point of dilation is the resolution of symptoms. Solid food dysphagia occurs with an esophageal lumen less than $13 \mathrm{~mm}$. In general, dilation up to $18 \mathrm{~mm}(54 \mathrm{Fr})$ will allow a regular diet to be consumed. In reality though, many patients will be able to eat a fairly normal diet after the passage of a $16 \mathrm{~mm}$ ( $48 \mathrm{Fr}$ ) dilator. It is important to instruct patients to chew their food well until they are dilated up to at least $16 \mathrm{~mm}$.

One of the myths regarding esophageal dilation pertains to a theoretical risk of perforation when the esophagus is biopsied before dilation by creating a 'break' in the mucosa on which the tip of a dilator could catch. As a result, some have recommended that biopsies should be performed after dilation. This is not necessarily practical, because this requires reinsertion of the endoscope in a patient that has just undergone esophageal dilation and may be restless and uncomfortable. There has been one series of 48 patients reported where 
esophageal dilation was performed immediately following esophageal biopsy and there were no perforations related to the biopsies (20). It is probably no more unsafe to perform dilation after biopsy than to attempt reintubation of the esophagus in a patient who has just undergone dilation where they may not tolerate reintubation very well. TTS balloon dilation would eliminate this controversy because the esophagus could be biopsied after dilation while the endoscope is still inserted. A prospective clinical trial comparing biopsy before and after dilation would be needed to really clarify this issue.

Food bolus impaction may be the initial presentation of a patient with an esophageal stricture. With prolonged impaction, the food bolus may cause significant damage to the esophageal mucosa with ischemia and even perforation of the esophagus. This raises the question of whether to dilate the esophagus after removal of the esophageal obstruction during the same session. With a short period of obstruction and only mild edema or inflammation seen endoscopically after removal of the food bolus, esophageal dilation can be performed safely (21). If the food bolus obstruction has been relieved by gently

\section{REFERENCES}

1. Marks RD, Richter JE. Peptic strictures of the esophagus. Am J Gastroenterol 1993;88:1160-73.

2. Pera M, Cameron AJ, Trastek VF, Carpenter HA, Zinmeister AR. Increasing incidence of adenocarcinoma of the esophagus and esophagogastric junction. Gastroenterology 1993;104:510-3.

3. Abele JE. The physics of esophageal dilatation. Hepatogastroenterology 1992;39:486-9.

4. Hirota WK, Petersen K, Baron TH, et al. Guidelines for antibiotic prophylaxis for GI endoscopy. Gastrointest Endosc 2003;58:475-82.

5. Nelson DB, Sanderson SJ, Azar MM. Bacteremia with esophageal dilation. Gastrointest Endosc 1998;48:563-7.

6. Wesdrop IC, Bartelsman JF, den Hartog Jager FC, Huibregtse K, Tytgat GN. Results of conservative treatment of benign esophageal strictures: A follow-up study in 100 patients. Gastroenterology 1982;82:487-93.

7. Kochhar R, Makharia GK. Usefulness of intralesional triamcinolone in treatment of benign esophageal strictures. Gastrointest Endosc 2002;56:829-34.

8. Repici A, Conio M, De Angelis C, et al. Temporary placement of an expandable polyester silicone-covered stent for treatment of refractory benign esophageal strictures. Gastrointest Endosc 2004;60:513-9.

9. Hernandez LJ, Jacobson JW, Harris MS. Comparison among the perforation rates of Maloney, balloon, and savary dilation of esophageal strictures. Gastrointest Endosc 2000;51:460-2. (Erratum in 2003;58:642).

10. Kozarek RA, Patterson DJ, Ball TJ, et al. Esophageal dilation can be done safely using selective fluoroscopy and single dilating sessions. J Clin Gastroenterol 1995;20:184-8.

11. Ho SB, Cass O, Katsman RJ, et al. Fluoroscopy is not necessary for Maloney dilation of chronic esophageal strictures. Gastrointest Endosc 1995;41:11-4. pushing the bolus into the stomach with the endoscope, this technique itself may dilate the stricture and further dilation may not be required during that session. However, the patient should be brought back to reassess the stricture endoscopically and further dilation can be performed at that time if needed. If the food bolus is removed orally, the stricture may need to be dilated during the same session to prevent another obstruction before follow-up endoscopy. If the esophageal mucosa looks acutely inflamed or ischemic, dilation should be deferred to another session after healing of the mucosa because, in my opinion, the risk of perforation with dilation may be increased.

\section{SUMMARY}

Esophageal dilation of benign strictures is both a challenging and rewarding procedure for the endoscopist. Patience and experience are as important, if not more so, than technical ability and newer equipment, in terms of efficacy and minimizing complications. Important questions about the procedure remain unanswered, providing opportunities for further investigation in well-designed trials.

12. Kadakia SC, Cohan CF, Starnes EC. Esophageal dilation with polyvinyl bougies using a guidewire with markings without the aid of fluoroscopy. Gastrointest Endosc 1991;37:183-7.

13. Pereira-Lima JC, Ramires RP, Zamin I Jr, Cassal AP, Marroni CA, Mattos AA. Endoscopic dilation of benign esophageal strictures: Report of 1043 procedures. Am J Gastroenterol 1999;94:1497-501.

14. Tulman AB, Boyce HW Jr. Complications of esophageal dilation and guidelines for their prevention. Gastrointest Endosc 1981;27:229-34.

15. Spechler SJ. AGA technical review on treatment of patients with dysphagia caused by benign disorders of the distal esophagus. Gastroenterology 1999;117:233-54.

16. Patterson DJ, Graham DY, Smith JL, et al. Natural history of benign esophageal stricture treated by dilatation. Gastroenterology 1983;85:346-50.

17. Glick ME. Clinical course of esophageal stricture managed by bougienage. Dig Dis Sci 1982;27:884-8.

18. Marks RD, Richter JE, Rizzo J, et al. Omeprazole versus $\mathrm{H}_{2}$ receptor antagonists in treating patients with peptic stricture and esophagitis. Gastroenterology 1994;106:907-15.

19. Saeed ZA, Winchester CB, Ferro PS, Michaletz PA, Schwartz JT, Graham DY. Prospective randomized comparison of polyvinyl bougies and through-the-scope balloons for dilation of peptic strictures of the esophagus. Gastrointest Endosc 1995;41:189-95.

20. Barkin JS, Taub S, Rogers AI. The safety of combined endoscopy, biopsy and dilation in esophageal strictures. Am J Gastroenterol 1981;76:23-6.

21. Weinstock LB, Shatz BA, Thyssen SE. Esophageal food bolus obstruction: Evaluation of extraction and modified push techniques in 75 cases. Endoscopy 1999;31:421-5. 


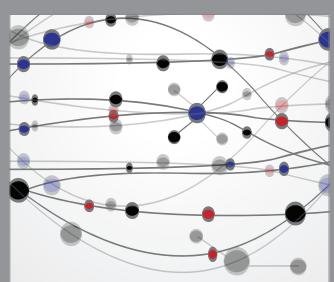

The Scientific World Journal
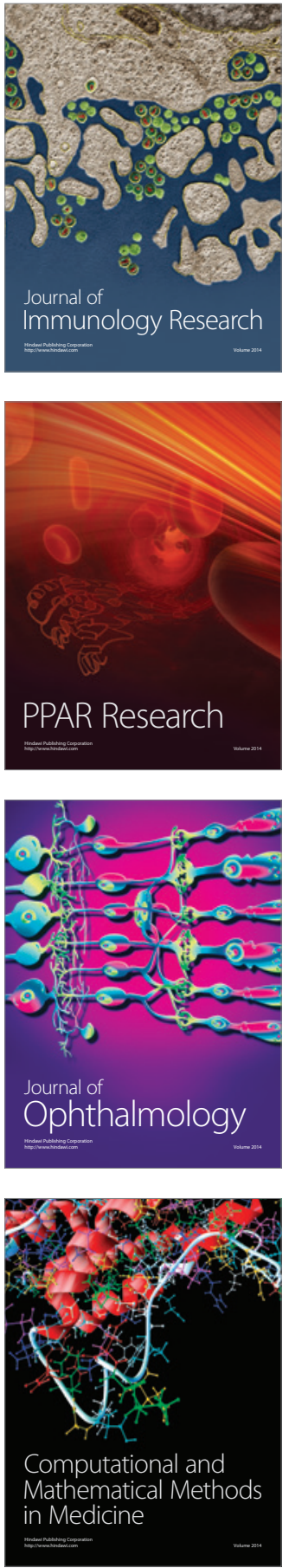

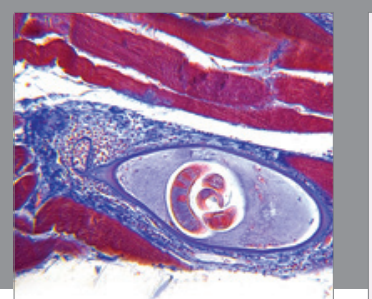

Gastroenterology Research and Practice

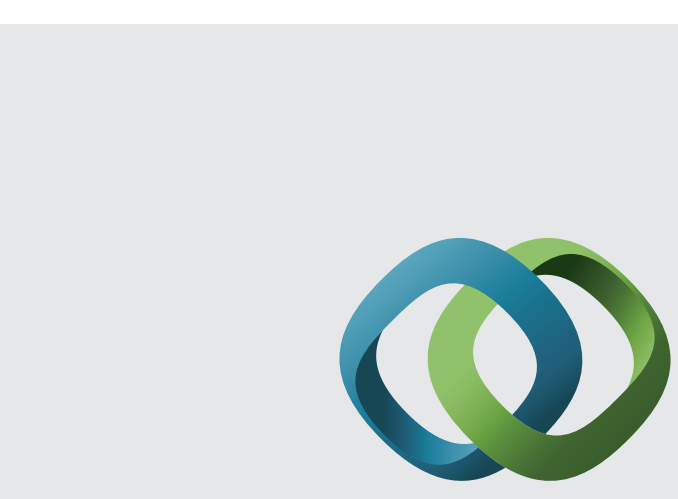

\section{Hindawi}

Submit your manuscripts at

http://www.hindawi.com
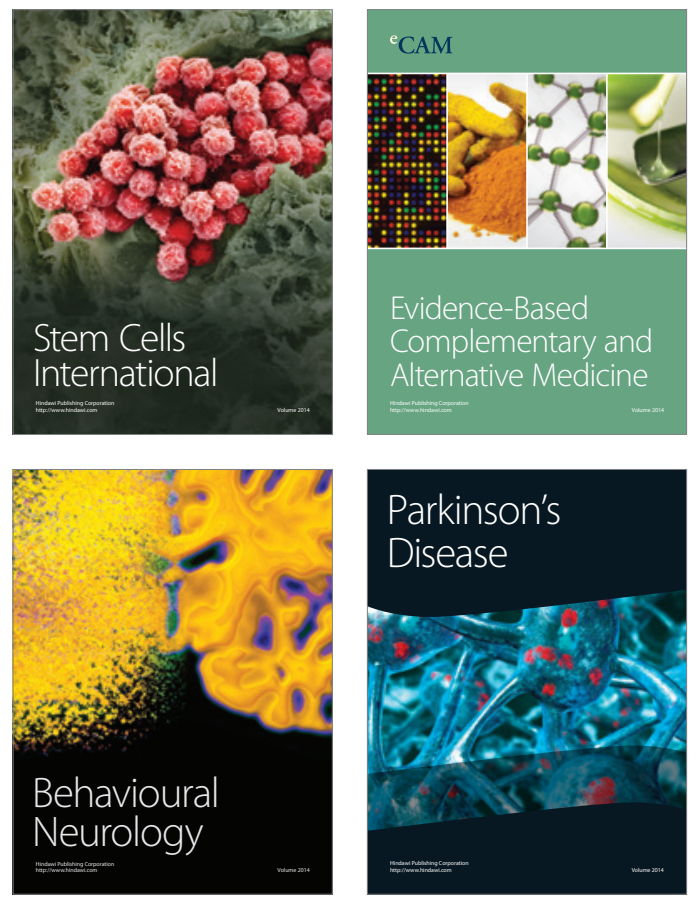
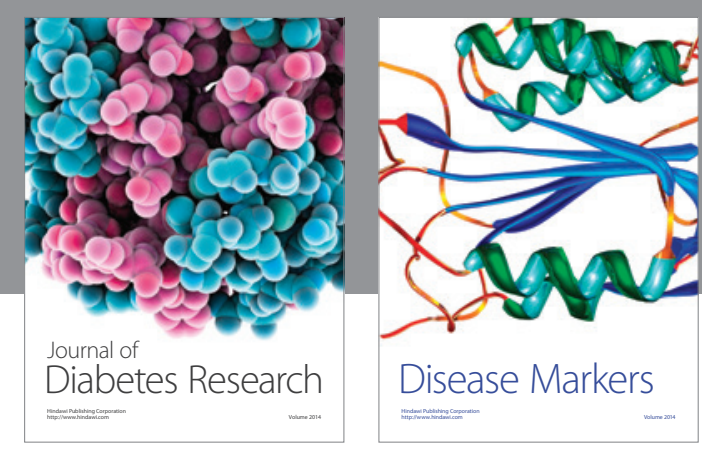

Disease Markers
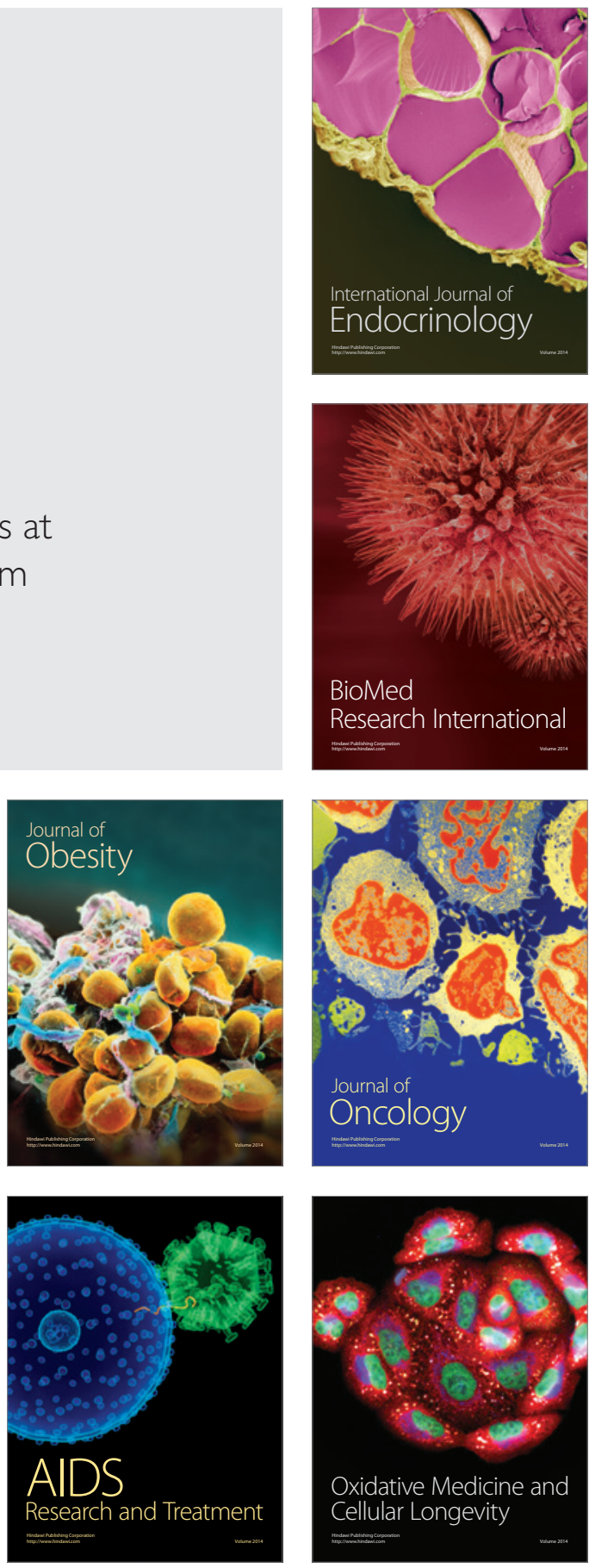